\title{
El italiano aplicado al canto como destreza
}

\author{
Ana Isabel Fernández Valbuena
}

\begin{abstract}
This paper attempts to shed some light on the specific teaching of Italian language applied to singing. We must admit its increasing demand on most Lyrical schools or $\mathrm{H}$ igh Schools of M usic all over the world.

$\mathrm{N}$ eedless to say there are no proper teacher-training centers to help teachers acquire and develop new methods and techniques on teaching languages for musical purposes. All other fields in language teaching are fully covered with ample research on every specific subject: economics, engineering, medicine, law, etc. As far as music is concerned, there is a lack of investigation on the linguistic abilities, viz. phonology and phonetics, a prospective singer will need when performing an aria in an opera or in an oratorio.

Up to now, our teaching activity is only based on our own experience. In this essay we propose a methological approach in order to provide an academic frame to the teaching of I talian language applied to singing.

And this is what we have been doing for several years at the «Escuela Superior de Canto» in M adrid.
\end{abstract}

La asignatura Ilamada «L engua italiana aplicada al C anto» forma parte desde hace años del programa de estudios de la Escuela Superior de Canto de M adrid, junto a la enseñanza de otros tres idiomas aplicados al canto: el alemán, el francés y el inglés. La importancia del estudio de estos idiomas para la carrera de un cantante está fuera de toda duda pues el repertorio que el cantante tendrá que abordar a lo largo de su quehacer profesional comprenderá, probablemente, piezas en estos idiomas, que tendrá que ejecutar con perfecta pronunciación. Esto ha abierto una demanda de profesores de una asignatura que se impartía de forma oficial en algunos conservatorios y de forma extraoficial en al gunos teatros líricos.

La titulación que el M inisterio de Educación ha exigido a los profesores de las lenguas aplicadas al canto ha sido la de Licenciados en Filología, que debe capacitar para el ejercicio de la docencia en lenguas extranjeras. Pero la asignatura a la que nos referimos exige una formación más específica, que ponga al filólogo en condiciones de comprender las necesidades de un alumno de Canto y la especial destreza que debe ejercitar en él. El profesor debería:

- Poseer un sólido conocimiento de la fonética de la lengua hablada, para poder desarrollar la percepción de la fonética cantada. 
- Saber desarrollar en el alumno los aspectos fonológicos de la lengua por encima de los gramaticales, pero, naturalmente, sin prescindir de ellos y, al tiempo, familiarizarlo con un léxico y una sintaxis diacrónicos que serán exclusivos de la lengua de los libretos.

- Debería conocer aproximadamente las diferencias del repertorio de la música vocal a lo largo de la historia y sus distintas implicaciones lingüísticas.

- D ebería conocer los rudimentos de la teoría de la música para afrontar los posibles problemas fonéticos que la ejecución de una partitura puede presentar y colaborar con el maestro de repertorio en la explicación integral de la pieza al alumno.

T odas estas destrezas sólo se adquieren de dos maneras: con el ejercicio continuado de la docencia, o a través de uno o varios cursos de adaptación a las nuevas especialidades.

La evolución de la glotodidáctica en los últimos años ha sido enorme y ha proporcionado numerosa y excelente bibliografía sobre la enseñanza de las lenguas extranjeras; se ha profundizado en las metas y los medios de la educación lingüística, se ha diversificado según el tipo de alumnos a los que van dirigidas estas enseñanzas y, sin embargo, en lo que se refiere al italiano aplicado al canto no conocemos que se haya hecho ninguna investigación glotodidáctica específica. La enseñanza sistematizada del italiano aplicado al canto es un reto que aún no ha sido abordado como campo de investigación con seriedad, ni en Italia, ni fuera de dicho país. D e entre la numerosa bibliografía glotodidáctica manejada la única referencia encontrada respecto a esta materia corresponde a Paolo Balboni: ${ }^{1}$

Eppure essa è insegnata in moltissimi conservatori musicali, dove se ne usa una varietà collocata tra il C inquecento el' 0 ttocento - un italiano incomprensibile per moltissimi parlanti nativi di italiano del ventesimo secolo.

Tale lingua, rientra ancora nella categoria dell'italiano come lingua moderna? Forse si tratta di una varietà che si potrebbe collocare tra le lingue classiche: il corpus testuale è finito: non c'è evoluzione; la produzione orale non esiste in quanto autonoma ma solo legata alla musica - che preme sulla musica, la deforma, la rende quasi incomprensibile.

$\mathrm{N}$ on diamo una risposta, perché c'è la necessità di un ulteriore approfondimento teorico. M olto di quanto si dirà in questo studio sarà solo parzialmente applicabile all'insegnamento dell'italiano della musica e del melodramma italiani, che richiede prospettive glottodidattiche proprie.

C reemos, pues, necesario dar rango de disciplina a esta materia, pero conviene advertir que lo que aquí vamos a tratar es un campo abierto a la investigación, desde la experiencia de al gunos años dedicados a la enseñanza de esta destreza del italiano, sin establecer dogmas. 
Tendríamos, en primer lugar, que intentar definir la asignatura, es decir, enumerar aquellas características que la hacen distinta de cualquier lengua que se estudia con fines exclusivamente comunicativos:

- La producción operística y de concierto italiana es extensísima, abarca casi cuatro siglos y tiene una historia propia que debe ser tratada en cada uno de sus momentos: el estilo musical de cada época ejerce una influencia directa sobre el texto y viceversa. Encontramos ocasiones en las que la fusión entre palabra y música es casi perfecta - como las primeras óperas del '600- y otras en que el texto está al servicio de la música - como en algunas óperas rossinianas.

- 0 tro aspecto importante, relacionado con la inteligibilidad del texto, es la estructura musical de la obra, que influye directamente en su relación con la palabra; no es lo mismo cantar un recitati vo secco casi sin apoyo musical, que un recitativo accompagnato, que un duo o un aria con acompañamiento, o que un concertante, en el que cantan simultáneamente varios intérpretes, donde es prácticamente imposible, entender lo que cada personaje está diciendo.

- U na tercera característica a considerar es la tesitura de las voces, a efectos de inteligibilidad del texto: las voces masculinas, por su timbre, se prestan más a la comprensión articulatoria que las femeninas y dentro de las femeninas más las graves que las agudas.

Por todos estos motivos - y sin entrar en la polémica secular sobre un arte "storpiata in servizio dell'altra" - quizá será más oportuno soslayar el término dependencia del texto a la música y hablar de interdependencia, constante de la que no podremos prescindir nunca ni desde el punto de vista teórico, ni desde el punto de vista metodológico. Texto y música avanzan en recíproca correspondencia, formando a veces un conjunto de gran belleza.

D ado que el corpus lingüístico con el que trabajamos no evoluciona y no existe producción oral, como apuntaba Balboni, debemos plantearnos las necesidades y objetivos del italiano cantabile: ¿qué tipo de competencia hay que desarrollar en estos alumnos? y como consecuencia ¿cual sería el método adecuado?

$\mathrm{D}$ ado que en estos momentos el concepto de necesidad ha adquirido un matiz más dinámico en el plano pedagógico y que se tiende a conectarlo más con la actividad que el alumno tendrá que desarrollar en la lengua motivo de estudio, que exclusivamente con el tipo de lengua que tiene que aprender, nuestro planteamiento como glotodidactas sería: ¿qué tiene que hacer un cantante cantando en italiano sobre un escenario?, en vez de ¿qué tipo de italiano hay que enseñar a un cantante?. La función de un cantante globalmente considerada sería: saber transmitir a un público que le está escuchando cualquier fragmento, ópera completa, aria, canción de concierto etc... de esa enorme producción del italiano de la música, de forma coherente y con una pronunciación correcta. 
Sus necesidades entonces serían: conseguir el domino del sistema fonético, fonológi co y gráfico de la lengua italiana, muy por encima de los aspectos gramaticales y comunicativos. En segundo lugar, lograr la transmisión de ese texto que canta dándole a la lengua su auténtica dimensión, que se consigue sólo comprendiendo lo que se canta, transformándolo en un verdadero acto de comunicación. De ese modo el texto cantado vuelve a adquirir categoría de lengua viva; de lo contrario, cuando es sólo una reproducción mecánica, habría que incluirla, como apuntaba Balboni, en la categoría de lengua clásica, es decir, muerta.

En lo que se refiere a la metodología del italiano aplicado al canto, moviéndonos dentro de la enseñanza de una $L 2$, utilizamos planteamientos generales, métodos y materiales que están a disposición de cualquier glotodidacta; pero ya desde el primer momento en que se aborda la materia resulta inoperante seguir la organización de los contenidos en el orden establecido en el método. N uestra trayectoria es un continuo alterar las normas básicas de la lingüística aplicada, para llevar al alumno caminando en paralelo, a alcanzar las necesidades de las que ya hemos hablado. $\mathrm{N}$ o se puede progresar en orden de dificultad de uso comunicativo, ni de frecuencia léxica, ni gramatical, puesto que desde el comienzo el alumno entra en contacto con un registro lingüístico áulico, del que se debe convertir en productor-transmisor.

Podríamos pensar que en el caso de un cantante que aborda el estudio de un idioma aplicado al canto se podrían descartar las destrezas productivas hablar y escribir y trabajar sólo con las receptivas: comprender y leer. Pero esto no es exacto, puesto que existe en estos alumnos una parte de producción; no completa, no como se concibe la producción en didáctica, porque lo que se elimina en su totalidad es la función interaccional - no hay interlocutor- , luego las dificultades de tener que comprender y comunicarse con otra persona no existen. La producción del cantante se fundamenta en lo que los lingüistas Ilaman "función transaccional", es decir, transmisión de información, que tiene características similares a las de la lengua escrita, aunque no idénticas: todo está previamente planeado, tiene mayor grado de formalidad que la lengua conversacional, en la forma hay abundancia de monólogos y carece de la espontaneidad y redundancia que caracteriza a la lengua hablada. Este tipo de producción constituye un continuum entre la escrita y la producción hablada; luego lo único que verdaderamente se puede eliminar es la destreza productiva escrita.

En cuanto al corpus textual es cerrado pero muy extenso: empieza aproximadamente en 1600 y coincide con las distintas etapas artísticas de la historia de la literatura y de la música. Tiene la sintaxis propia del verso y el léxico es arcaizante. Pero el alumno que se enfrenta a este tipo de repertorio tiene que trabajarlo de forma activa y este es el punto más significativo y el mayor reto de la asignatura: hacer trabajar al alumno de una manera activa con un corpus que poco tiene que ver con lo que es la lengua tal y como hoy se concibe (en función de su uso).

La lengua de los libretos - la canción de concierto tiene otro trata- 
miento- fuera de su contexto melodramático no tiene hoy en día ningún uso y separada de la música carece a menudo de valor artístico; luego el libreto no puede presentarse nunca como modelo de comunicación, porque, además, su registro resulta muy difícil para una persona de competencia relativa en italiano. Es necesario, por lo tanto, crear una vía de acceso a este corpus y la única posibilidad es partir de la lengua de hoy, de la sincronía y no únicamente, sino además de la lengua tal y como se estructura para enseñarse; es el mejor modo de ejercitar la producción oral. A esta conclusión hemos llegado basándonos en una postura que defienden muchos teóricos como K rashen ${ }^{2}$, uno de los que más ha contribuido a la investigación en este campo pedagógico y que afirma: «La lingua che non è capita non è nemmeno appresa».

Partir del italiano normativo de hoy facilita al alumno el camino para producir, al principio, mensajes (imput) comprensibles; permite además trabajar la fonética no como una práctica articulatoria vacía de contenido, sino como una parte de la lengua que no puede aislarse del resto de las partes que la componen. Este punto de partida nos permite también trabajar de una manera activa con la lengua, produciendo y comprendiendo formas gramatical y sintácticamente correctas, que al constituir la estructura de la lengua, son comunes a la lengua de hoy y a la de los libretos. A su vez, el progreso adecuado de las estructuras gramaticales y sintácticas permite el acceso a la sintaxis y al léxico propios de la libretística, que sólo se llegan a comprender tomando como punto de referencia las formas de la lengua viva.

D e modo que la propuesta de trabajo se basa en un método mixto, que comprende ejercicios de un método comunicativo, basado en la audición de conversaciones en italiano y en la producción oral fundamentalmente, con el apoyo de un libro de texto; al tiempo el alumno comienza su acercamiento al registro melodramático mediante las piezas vocales que debe cantar en italiano y que prepara, desde el punto de vista lingüístico y fonológico con el profesor. Paralelamente se comienza en el aula la lectura de un libreto en grupo, sobre el que se va trabajando la diacronía y se insiste sobre los fonemas. En todos estos ejercicios la corrección fonética es el objetivo primordial.

Conscientes de que la sensibilidad y la apreciación del hecho artístico es algo que se adquiere con el tiempo, que se va educando lentamente, aceptamos la necesidad de acometer de manera más directa la comprensión del texto que se canta como si se tratase de una materia técnica. En estos casos el conocimiento activo de la lengua se basa en un vocabulario "operativo", es decir, en el de los elementos no-técnicos que ligan el discurso y el estudio se centra en la lengua escrita, sobre un léxico concreto, fácilmente identificable: el lenguaje poético en este caso. La comprensión del texto antiguo favorece la correcta interpretación del mismo y capacita al alumno para introducir medios prosódicos y mímicos como recurso expresivo. 
Partiendo de estos presupuestos e insistiendo en el hecho de que la metodología que se propone es puramente empírica y de que su sistematización y su adecuado material didáctico están aún por hacer, exponemos sumariamente el desarrollo de los contenidos de la asignatura, tal como se ha estructurado en los últimos años en la Escuela Superior de C anto de M adrid.

El profesor desarrolla su actividad docente en tres módulos distintos:

A) Clases de grupo (máximo 15 alumnos).

B) Clases individuales.

C) Clases individuales con el repertorista - pianista acompañante- de idiomas.

C ada módulo cubre unos objetivos específicos, pero ninguno de ellos tendría sentido por sí solo porque son interdependientes.

\section{A) C lases de grupo}

O peran sobre los dos niveles lingüísticos mencionados: italiano normativo sincrónico y prácticas de lectura del italiano del repertorio vocal, desde el siglo XVII hasta nuestros días. En estas clases de grupo se sientan las bases fonológicas de la lengua que se aprende y aunque el didacta opera con la fonética como con el resto del material, siguiendo los criterios de presentación graduada, análisis contrastivo, variaciones al ofónicas, etc., es evidente la prioridad absoluta del aspecto fonológico sobre todos los demás. La insistencia en la reproducción exacta primero y en la producción exacta después, de los fonemas, en los distintos contextos, se debe reiterar en cada alumno y en cada clase de grupo.

El modelo didáctico el egido para la ejercitación de la lengua del libreto es la lectura en común de uno de estos textos, más breve y sencillo en el primer curso, más complejo en registro y argumento en el segundo. El ejercicio se plantea de forma distinta según el nivel:

Nivel 1

- Se enfoca la lectura a la correcta emisión de los fonemas en la cadena hablada, familiarizando al alumno con sonidos que en italiano actual son menos comunes (fonética diacrónica).

- Se realizan los comentarios morfológicos oportunos que distinguen esta variedad de lengua y se explican los arcaísmos y su moderna acepción, exponiendo al alumno el significado global del texto que se ha leído - y cuya comprensión por parte del alumno rara vez alcanza el $60 \%$.

Nivel 2

- En la lectura se trabaja no sólo la articulación correcta sino también los rasgos prosódicos como ritmo, acento y entonación, cuya adquisición es una cuestión de oído y de costumbre. 
- El trabajo sobre las estructuras alcanza el nivel sintáctico, ya que su organización en la lengua poética nada tiene que ver con la de la lengua hablada: frecuentes hipérbaton, repeticiones...

- D e la comprensión global el alumno puede acercarse a la traducción, con el auxilio del profesor.

La elección de un libreto único, en lugar de textos de distintas piezas vocales como práctica de lectura en grupo no es arbitraria: se trata de un texto con un argumento, que se explica al comienzo de la lectura, ayudando al proceso de comprensión; la mayoría de los libretos contienen recitativos, más cercanos al uso de la lengua hablada que el código de las arias y al tratarse de un texto completo el discente consigue, a lo largo del curso, una visión de conjunto.

\section{B) C lases individuales}

En encuentros paralel os a las clases de grupo se trabajan de forma individualizada los textos de las piezas que cada discente debe cantar. El proceso que se sigue con cada partitura es minucioso y lento:

- Se lee el texto completo para comprobar si el alumno ha entendido dónde comienza cada palabra y cada frase, dado que en la partitura el texto aparece dividido en sílabas, y que las sílabas de cada palabra suelen unirse entre sí a través de guiones.

- Se corrigen los eventuales errores de pronunciación y si se detectaran dificultades fonéticas reiteradas se propone al alumno algún ejercicio articulatorio, para superarlas.

- Por último, el profesor comprueba si el alumno conoce el argumento de la obra en la que se incluye la pieza - en el caso de que se trate de un fragmento-, traduce el texto, implicando al alumno más aventajado y explica el código retórico en el que se enmarca dicho lenguaje, para que el futuro intérprete comprenda su verdadera dimensión y pueda, a su vez, comunicarla.

\section{C) Clases individuales con repertorista}

En este punto se encuentran el profesor de lengua, el profesor que acompaña al piano y el alumno y en él converge todo lo aprendido durante las clases en grupo y las de lectura individual; el alumno hace aquello para lo que se le prepara: CAN TA. Puede parecer una afirmación de perogrullo, pero queremos insistir en ello porque tal como se está orientando la asignatura en los conservatorios de Grado medio en España este momento fundamental no se está contemplando. Es decir, el profesor de lengua aplicada al canto prepara al alumno para la ejecución de algo que el profesor nunca le verá hacer, al menos en el contexto del aula.

El docente debe escuchar CAN TAR al alumno, controlando las posibles deficiencias articulatorias; es más, el italiano cantado debe ser la parte funda- 
mental, a nuestro juicio, de la evaluación final de la asignatura en cada alumno. Sucede a menudo que estudiantes con aceptable dicción en la lectura presentan problemas articulatorios al cantar; o viceversa, hay personas con cierta dureza articulatoria o entonativa en la lectura, a las que el canto ayuda a superar los escollos, facilitando su ejecución fonética. No debemos olvidar que se trata de emitir lengua cantada, cuya articulación debe ser no sólo correcta, sino delicadamente exagerada para que en el canal emisor no se pierdan los matices.

Para concluir sólo nos resta insistir en la necesidad de formación e información en una materia que, aunque ya goza de una cierta tradición, experimental y restringida, está aumentando debido a su reciente implantación. 